\title{
Erratum to: Role of the Renin-Angiotensin-Aldosterone System and Its Pharmacological Inhibitors in Cardiovascular Diseases: Complex and Critical Issues
}

\author{
Claudio Borghi $^{1} \cdot$ SIIA Task Force $\cdot$ Francesco Rossi $^{2} \cdot$ SIF Task Force
}

Published online: 4 January 2016

(C) Springer International Publishing Switzerland 2015

\section{Erratum to: High Blood Press Cardiovasc Prev DOI 10.1007/s40292-015-0120-5}

In the text: the word "pre-load" (page 5) must be replaced by the word "post-load".

The correct affiliation of the author Francesco Rossi is: Department of Experimental Medicine, Second University of Naples, Naples, Italy

The online version of the original article can be found under doi:10.1007/s40292-015-0120-5.

Claudio Borghi

claudio.borghi@unibo.it

1 Chair of Internal Medicine, Department of Medical and Surgical Sciences, Division of Internal Medicine, Ospedale Policlinico Sant' Orsola-Malpighi, University of Bologna, Via Albertoni, 15, 40138 Bologna, Italy

2 Department of Experimental Medicine, Second University of Naples, Naples, Italy 